\title{
POSITRON ANNIHILATION IN AND COMPRESSIBILITY OF WATER-ORGANIC MIXTURES. THE SYSTEM WATER-ACETAMIDE
}

\author{
K. JERIE*, A. BARANOWSKI \\ Institute of Experimental Physics, University of Wrocław \\ Cybulskiego 36, 50-205 Wrocław, Poland \\ J. GlińsKi and K. ORzechowsKi \\ Faculty of Chemistry, University of Wrocław \\ F. Joliot-Curie 14, 50-383 Wrocław, Poland \\ (Received April 4, 1996; revised version June 26, 1996; \\ in final form August 26, 1996)
}

\begin{abstract}
The experimental results of positron annihilation experiments in aqueous solutions of acetamide were compared to those of ultrasonic velocity. The system under investigation behaves untypically when changing acetamide concentration. From the point of view of surface tension it interacts with water like normal alcohols, while the positron annihilation and ultrasonic results suggest hydrophilic hydration and lack of hydrophobic interactions.
\end{abstract}

PACS numbers: $61.25 . \mathrm{Em}, 78.70 . \mathrm{Bj}$

\section{Introduction}

In the papers of this series we investigated the structure of aqueous solutions of nonelectrolytes: tetrahydrofuran [1], cyclohexylamine [2], methyl alcohol [3], ethyl alcohol [4], $n$-propyl alcohol [5], isopropyl alcohol [6], dimethylsulfoxide [7], and pyridine [8]. As far, however, all the solutes investigated had hydrophobic parts, and their interactions with water framework could be explained in terms of hydrophobic hydration $[9,10]$. Encouraged by some success in comparing two independent experimental methods, the positron annihilation and the ultrasonic velocity, we try now to test the title system, where no hydrophobic hydration should occur and any interactions should have hydrophilic character only.

It is obvious from the results of our previous positron annihilation experiments that all the systems investigated exhibit characteristic dependences of annihilation parameters on concentration of nonelectrolyte in water. In all the systems

\footnotetext{
*Corresponding author.
} 
their spectra could be resolved into at least two components having different lifetimes. They can be interpreted assuming many possible mechanisms of positronium annihilation in liquid. The long-lived component of the spectrum corresponds to ortho-positronium annihilation in the pick-off process, while the short-lived one to free positron annihilation as well as that of para-positronium (and/or others). All the details of treatment of the annihilation data are given in detail in other papers of this series $[1,2,4,7]$. The resolution is proceeded with respect to the condition $I_{1}+I_{2}+I_{3}=100 \%$, where $I_{i}$ are the intensities of the components of the spectrum. However, only the long-lived component is of interest in interpretation, and its parameters remain almost undisturbed when changing the number of the components.

After the resolution, each component is described by two parameters: lifetime $\tau_{i}$ and intensity $I_{i}$. Formation as well as lifetime of ortho-positronium, which is responsible for the long-lived mechanism, is strongly influenced by the physical properties of the medium surrounding the positronium pseudoatom. The liquid water structure, with cavities and open hydrogen-bonded network, can be modified and then stabilized by presence of a guest molecule. The nonelectrolytes form strong hydrates of quasiclathrate architecture, where considerably big voids are occupied by solute molecules. Thus, the liquid structure should become more openwork, but also more rigid. The competition of these two effects yields characteristic concentration dependences of $\tau_{3}$ and $I_{3}$ : the former exhibits an inflection and the latter one - a maximum at the concentration corresponding to the stoichiometry of the hydrates formed - see our previous papers cited in this paper.

One can extract the same stoichiometry from ultrasound velocity data. From Laplace's equation $\beta=\left(\rho c^{2}\right)^{-1}$ the adiabatic compressibility coefficient $\beta$ can be calculated ( $\rho$ - density of liquid, $c$ - sound velocity). When plotting the adiabatic compressibilities $\beta$ against nonelectrolyte concentration in water, one observes that isotherms of $\beta$ intersect usually at a common point. The $X$-coordinate of this point gives stoichiometry of the quasiclathrate hydrates, according to the concept of Endo [11]. The details of this interpretation are given in [12] and [13]. It is worth noting that the compressibility value at the crossing point is, in most cases, very close to that found by von Stackelberg and Müller for the solid clathrates [14].

The series of our previous papers concerned the systems of aqueous solutions of nonelectrolytes, all of them (except water + methanol) containing well-defined hydrophobic part of the molecule, which allows the hydrophobic hydration. The two different experimental methods used, i.e. positron annihilation and sound velocity, yielded similar results, explainable in terms of structure-breaking concept. The stoichiometries of hypothetical quasiclathrate hydrates were similar, too, and independent on the method.

In this paper we investigated the system water + acetamide $\left(\mathrm{CH}_{3}-\mathrm{CO}-\mathrm{NH}_{2}\right)$. While the solute has no distinct hydrophobic part in its molecule, only hydrophilic hydration should be possible. It seemed interesting how differs the experimental picture of a system without hydrophobic hydration from those investigated before. 


\section{Experimental}

Chemicals. Acetamide (analytical grade) from POCh, Gliwice, Poland, was recrystallized from aqueous solution and dried (for surface tension measurements) or used without further purification (acoustical and annihilation experiments). Freshly prepared, double distilled water was used. Solutions were prepared by weighing.

Positron annihilation measurements. The annihilation measurements were carried out at room temperature (approximately $25^{\circ} \mathrm{C}$ ) with a standard measuring device based on the "fast-slow" coincidence technique as described elsewhere [1]. The obtained spectra were resolved using POSITRONFIT program.

Sound velocity measurements. The ultrasonic velocity was measured with an accuracy of $\pm 0.1 \mathrm{~m} / \mathrm{s}$ using a "sing-around" equipment (apparatus Ecolab MPFU, Poland). The details of the apparatus and measurement techniques applied in this study are given in Ref. [15].

Density measurements. Density was measured using a vibrating pipe method (apparatus Ecolab MG-2, Poland). The accuracy was not worse than $\pm 0.02 \mathrm{~kg} / \mathrm{m}^{3}$.

Surface tension measurements. Apparatus Krüss K12 was used operating with Wilhelmi platinum plate $20 \mathrm{~mm}$ long. The accuracy was about $\pm 0.0002 \mathrm{~N} / \mathrm{m}$.

\section{Experimental results}

Solubility of acetamide in water at room temperature is limited to mole fraction below 0.2 . While all the structural rearrangements of solution should occur below this concentration, this number seemed sufficient for our purposes.

Sound velocity. The adiabatic compressibility coefficients $\beta$ were calculated from Laplace's equation $\beta=\left(\rho c^{2}\right)^{-1}$ (where $\rho$ - density of the liquid, $c$ - sound velocity). The concentration dependence of the compressibility in the low nonelectrolyte concentration region, which is of interest for the further discussion, is shown in Fig. 1.

The adiabatic compressibility plotted against the mole fraction of acetamide (Fig. 1) shows that there is no common intersection point of isotherms of adiabatic compressibility. The isotherms intersect depending on temperature at mole fraction of the solute varying from 0.09 to 0.11 (in the temperature range studied). This result is different comparing with the known aqueous solutions of nonelectroly tes with hydrophobic group $[1,2,4-8]$ (where the shift of the intersection point was hardly observed) and similar to the system water-methanol [3].

Positron mean lifetimes. The numerical treatment briefly described above yielded the parameters of the annihilation spectrum resolved into three components: lifetimes $\tau_{1}, \tau_{2}, \tau_{3}$ and corresponding intensities: $I_{1}, I_{2}, I_{3}$. The concentration dependencies of the long-lived component's parameters $\left(\tau_{3}\right.$ and $\left.I_{3}\right)$ are shown in Figs. 2 and 3. For comparison, the corresponding results obtained for the system water + ethanol (a system with dominating hydrophobic hydration, where quasicrystalline clathrate-like hydrates of approximate stoichiometry Eth $17 \mathrm{H}_{2} \mathrm{O}$ are formed) are also shown in these figures.

Both $\tau_{3}$ and $I_{3}$ seem to change monotonically with acetamide concentration. It should be noted that both the short-lived lifetimes, $\tau_{1}$ and $\tau_{2}$, decrease with 


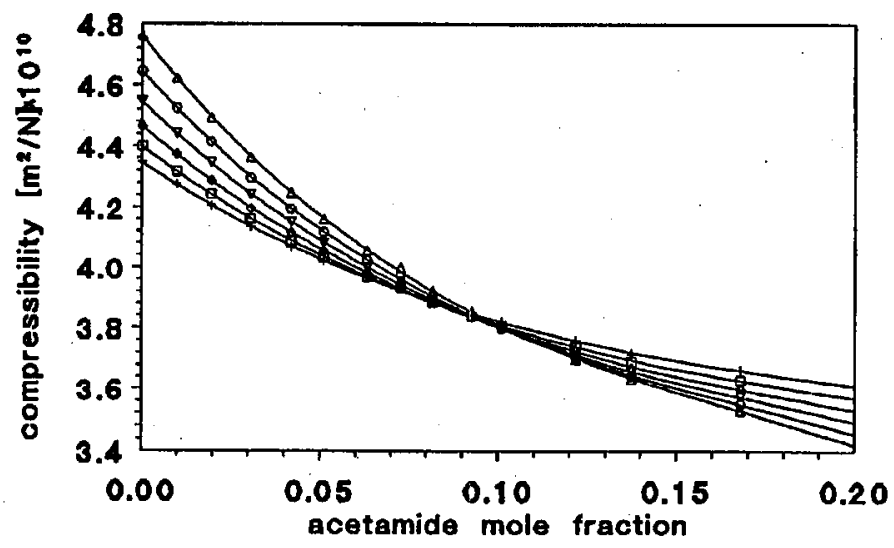

Fig. 1. Plots of adiabatic compressibility coefficients vs. mole fraction of acetamide in water. $\triangle 283 \mathrm{~K}, \bigcirc 288 \mathrm{~K}, \nabla 293 \mathrm{~K}, \diamond 298 \mathrm{~K}, \square 303 \mathrm{~K},+308 \mathrm{~K}$. Lines are drawn arbitrarily (spline function).

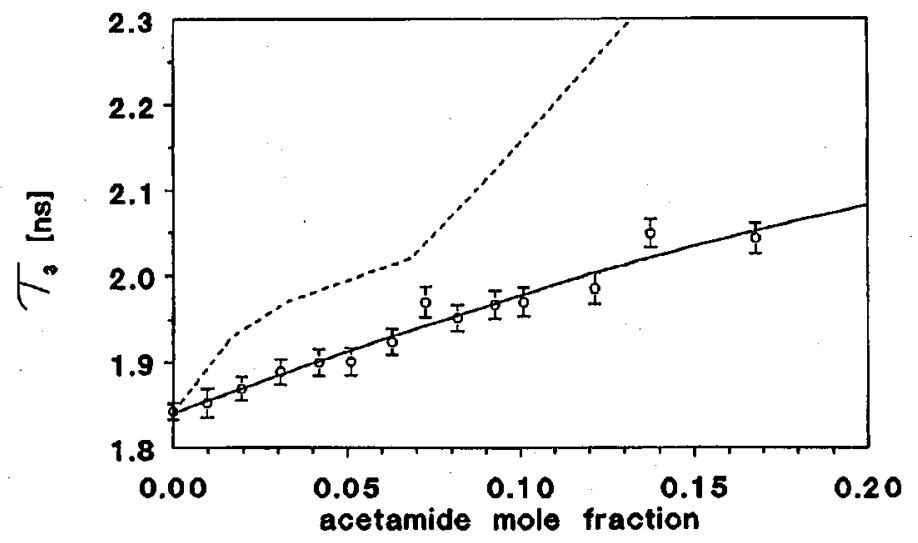

Fig. 2. Lifetimes of the long-lived component of annihilation spectra vs. acetamide concentration in water. Broken line: the results for aqueous solutions of ethanol [4].

addition of the solute, but their intensities are apparently changing inversely. This may be caused by not complete separation of the processes responsible for the short-lived annihilation. While their lifetimes are similar, the processes cannot be separated and the resulting parameters are complementing each other. In different words, the mechanisms of short-lived processes are too close one to another to be separated.

In the limits of the errors, the concentration dependence of $\tau_{3}$ in Fig. 2, as well as that of $I_{3}$ in Fig. 3, seems to be smooth. The annihilation results differ significantly from those obtained in the aqueous mixtures of normal alcohols and 


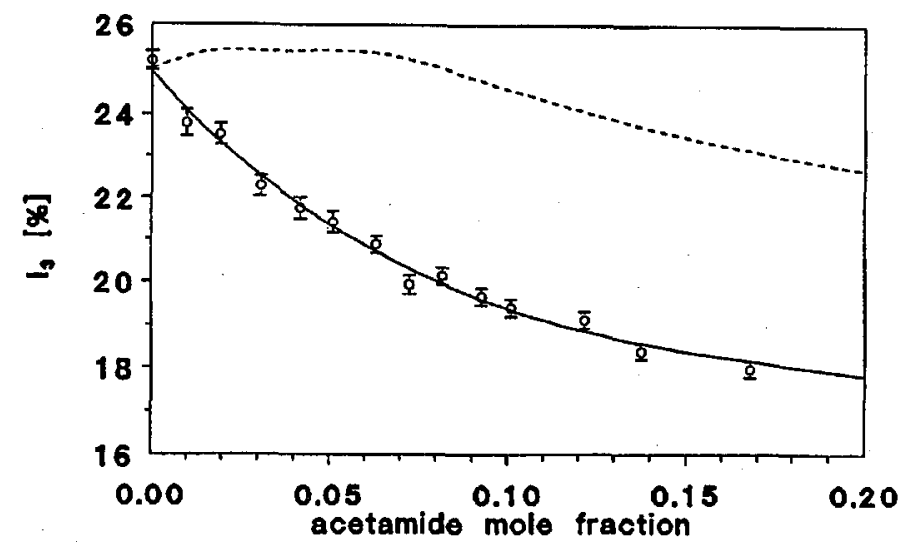

Fig. 3. Intensities of the long-lived component of annihilation spectra vs. acetamide concentration in water. Broken line: the results for aqueous solutions of ethanol [4].

other solutes with hydrophobic group. There is neither inflection of $\tau_{3}$ nor maximum of $I_{3}$, which could be observed in the hydrophobically hydrated systems at concentrations roughly corresponding to stoichiometries of liquid quasicrystalline hydrates (see broken lines in Figs. 2 and 3).

\section{Discussion}

The formation of clathrate hydrates in the aqueous solutions of nonelectroly tes with hydrophobic parts of their molecule is well proved now. According to the concept of iceberg formation of Frank and Evans [9] or to that of hydrophobic hydration formulated by Franks [10], when an organic compound is dissolved in water, the water structure is modified around the nonpolar parts of the solute molecule in the direction of greater "crystallinity", whatever the actual quasi-solid water structure induced in this way. In the vicinity of guest molecule the water structure is determined mainly by its mobility. Molecules of solute are accommodated in the cavities of the open hydrogen-bonded network, which in pure water are most probably occupied by "monomeric" water molecules [16-19].

The system under investigation is different. The lack of hydrophobic hydration means that acetamide molecules should be accommodated in the hydrogen-bonded structure of water rather than in its cavities. Such accommodation is possible in every concentration, thus one does not observe any distinguished region of changing structure, while the changes are continuous.

Let us stress that the systems where solute molecule has no hydrophobic group can be structure-breakers, like those with hydrophobic parts. Negative deviations in $\varepsilon$ vs. composition curves ( $\varepsilon$ - relative permittivity) reported by Corradini et al. [20] for the system water + ethane-1,2-diol is an example of the system where no hydrophobic hydration occurs. The authors interpreted their results in terms of formation of strong hydrogen bondings between diol and water molecules, as well as between molecules of the components. 
A good example of interpretational problems in the cases of water-nonelectrolyte systems (where the solute has no hydrophobic part) is the system water-urea. Recently, Boek and Briels [21] investigated it theoretically using molecular dynamics simulations and comparing the results with those from neutron scattering experiments performed by Finney and Turner [22]. In contrary to the previous models of Frank and Franks [23], Schellman [24] (refined by Kreschek and Scheraga [25]) and Stokes [26], Boek and Briels conclude that for water-urea system of considerably high urea concentration $(2 \mathrm{M})$, the presence of dimers is very improbable. Of course, this conclusion should be valid for lower concentrations, too.

Applying this result to the title system means that hydration of acetamide in aqueous solutions should not be similar to the solutions where strong hydrophobic hydration occurs. The structural changes are smooth, since the solute molecules are hydrogen-bonded with water network.

The concentration of acetamide corresponding to (temperature dependent) interception of compressibility isotherms, equal to $\approx 0.1$ mole fraction of acetamide, is close to that corresponding to formation of type I clathrates hydrates (like it was postulated, although not proved, for the system water-methanol [3]). It is interesting, however, that the value of compressibility at this concentration $\left(\beta \approx 39 \times 10^{-11} \mathrm{~m}^{2} / \mathrm{N}\right)$ is almost the same as for solid clathrate hydrates measured by von Stackelberg and Müller [14]. At first sight it seems reasonable to assume that at mole fraction of acetamide equal to $\approx 0.1$ the solution contains mainly a mixture of hydrated solute molecules of the average hydration number $\approx 9$.

The concentration dependences of positron annihilation parameters in Figs. 2 and 3 seems to confirm the above considerations. The lack of drastic structure changes results in the observed smooth dependencies, with no inflections and extremas. Similar character of the concentration dependence of annihilation parameters, as well as that of compressibility, was observed by us for the aqueous systems with ethylene glycol [27], formamide [28] and methanol [3]. However, detailed inspection of the results raises doubts.

Usually only the long-lived component's parameters, here denoted as $\tau_{3}$ and $I_{3}$, are interpreted in terms of liquid structure. According to the concept of Levay, Vertes, and Hautojarvi, for an ideal mixture of classical liquids the lifetime should be a simple function of concentration [29]:

$$
\tau_{3, A B}^{-1 / 2}=\left[\tau_{3, A}^{-1 / 2} V_{A}-\left(\tau_{3, A}^{-1 / 2} V_{A}-\tau_{3, B}^{-1 / 2} V_{B}\right) X_{B}\right] \frac{1}{V_{A B}},
$$

where subscripts $A, B$, and $A B$ denote pure components $A$ and $B$, and their mixture, respectively; $V$ is the molar volume. In the case of a mixture its mean molar volume $V_{A B}$ can be calculated from the molar volumes of the components: $V_{A B}=\left(X_{A} M_{A}+X_{B} M_{B}\right) / \rho(M-$ molecular mass $)$. However, the experimental values of $\tau_{3}$ differ significantly from those obtained from the above relation, therefore it is evidently incorrect for the aqueous solutions of acetamide. Another concept, known as the "bubble" model of pick-off annihilation [30], assumes that lifetime of ortho-positronium is determined primarily by surface tension of the liquid

$$
\tau_{3, A B}^{-1}=\alpha \sigma^{\gamma}
$$


where $\sigma-$ surface tension of liquid, $\alpha$ and $\gamma-$ empirical (fitted) parameters. The experimental surface tensions of water + acetamide mixtures (shown in Fig. 4) change with concentration of the organic component in the manner typical of surface-active substances ( $\sigma$ decreases rapidly reaching at $X_{\text {acetamide }} \approx 0.1$, a value apparently very close to that of pure solute).

The concentration dependence of surface tension of the system under test seems surprising. The molecule of acetamide is small and should, generally, interact with the solvent in a hydrophilic way. Nevertheless, Eq. (2) describes the observed relation between $\tau_{3}$ and $\sigma$ much better than Eq. (1) and will be exploited further.

While acetamide melts at $82^{\circ} \mathrm{C}(355 \mathrm{~K})$, we were not able to measure the surface tension of pure acetamide; the same concerns its annihilation behavior. It is reasonable, however, to extrapolate the concentration dependences of the respective parameters and to use these extrapolated values in further calculations. Thus, we assumed arbitrarily that for pure acetamide at $293 \mathrm{~K}$ its $\tau_{3}$ is approximately $2.1 \mathrm{~ns}$ (see Fig. 2), surface tension is approximately $0.051 \mathrm{~N} / \mathrm{m}$ (see Fig. 4) and density is $1030 \mathrm{~kg} \cdot \mathrm{m}^{-3}$. These numbers allowed us to use Eqs. (1) and (2) and to compare the "calculated" lifetimes with the experimental ones. The results are shown in Fig. 5.

Confrontation of this result with our previous ones (obtained for aqueous systems with prevailing hydrophobic hydration and cited before) clearly shows that the mixtures tested behave like the systems investigated before (aqueous solutions of ethanol, $n$-propanol, isopropanol etc.): the "bubble" model (Eq. (2)) describes satisfactorily the $\tau_{3}$ changes in the region of high solute concentrations. At low concentrations, where the liquid structure is being rebuilt, $\tau_{3}$ is always lower than that predicted by the model, indicating the increased local rigidity of the system and/or its. locally increased density (existence of aggregates).

The most surprising is, however, that the ultrasonic velocity and annihilation results are typical of the systems with hydrophilic hydration, while the surface tension data suggest a reverse situation. Consequently, it is not possible now to

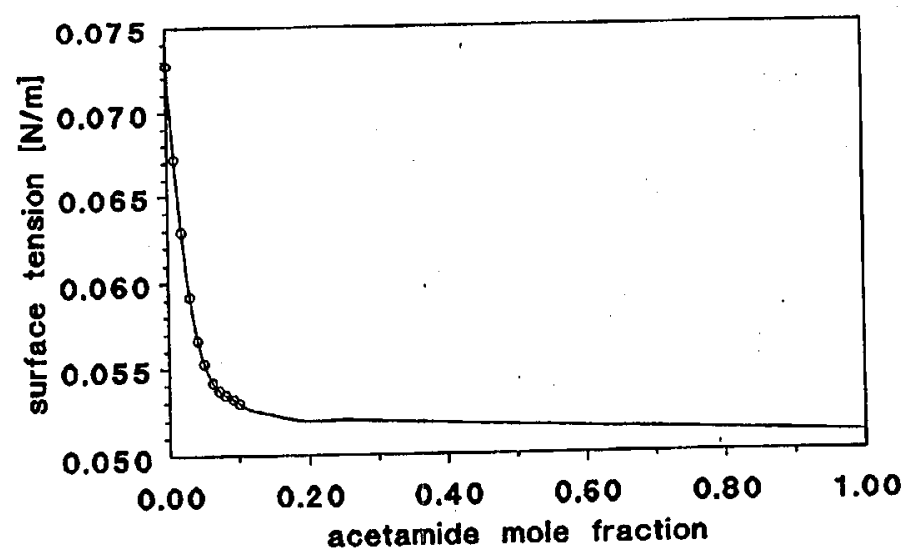

Fig. 4. Surface tension vs. acetamide concentration in water at $293 \mathrm{~K}$. 


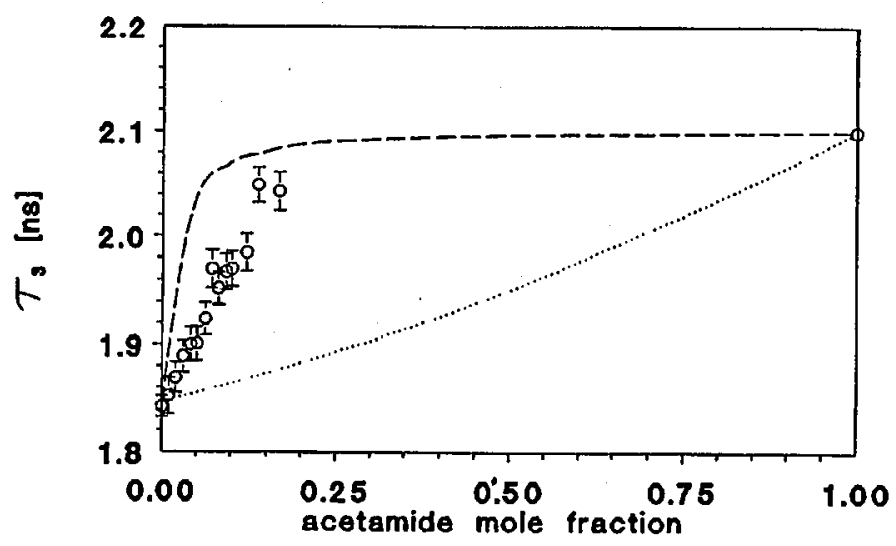

Fig. 5. The dependence of lifetime of the long-lived component of the annihilation spectrum $\tau_{3}$ vs. acetamide content in water at $293 \mathrm{~K}$. The dotted line was calculated according to Eq. (1) from molar volumes, the broken one was calculated according to the "bubble" model of the pick-off annihilation (Eq. (2)). The $\alpha$ and $\gamma$ parameters are $1.43 \times 10^{9}$ and 0.369 , respectively (see text).

interpret the structural rearrangements of the system under test. Obviously it needs further investigations involving other physical methods.

\section{Conclusions}

The acetamide molecule contains no hydrophobic part, which should imply gradual structural changes in solution when adding this solute to water. No clathrate-like hydrates are supposed to form, according to the ultrasonic results. The concentration dependence of surface tension suggests, however, that acetamide behaves like surface active agent. It seems impossible now to decide about the character of interactions of acetamide with water network.

\section{Acknowledgments}

This work is a part of the MEN GBW/95/5/IFD grant. A financial support from the Committee for Scientific Research is also acknowledged. One of us (J.G.) thanks Prof. J.-K. Platten (State University in Mons, Belgium) for the opportunity to measure the surface tensions of the system under investigation.

\section{References}

[1] K. Jerie, A. Baranowski, B. Rozenfeld, S. Ernst, J. Gliński, Acta Phys. Pol. A 64, 77 (1983).

[2] K. Jerie, A. Baranowski, B. Rozenfeld, B. Jeżowska-Trzebiatowska, S. Ernst, J. Gliński, Acta Phys. Pol. A 66, 3 (1984).

[3] K. Jerie, A. Baranowski, B. Rozenfeld, S. Ernst, J. Glinski, Acta Phys. Pol. A 66, 167 (1984). 
[4] K. Jerie, A. Baranowski, S. Ernst, J. Gliński, Acta Phys. Pol. A 69, 81 (1986).

[5] K. Jerie, A. Baranowski, S. Ernst, J. Gliński, Acta Phys. Pol. A 69, 91 (1986).

[6] K. Jerie, A. Baranowski, B. Rozenfeld, J. Gliński, S. Ernst, Acta Phys. Pol. A ì3, 667 (1988).

[7] K. Jerie, A. Baranowski, B. Rozenfeld, B. Jeżowska-Trzebiatowska, J. Gliński, Acta Phys. Pol. A 79, 507 (1991).

[8] K. Jerie, A. Baranowski, B. Rozenfeld, B. Jeżowska-Trzebiatowska, J. Gliński, Acta Phys. Pol. A 82, 183 (1992).

[9] H.S. Frank, M.W. Evans, J. Phys. Chem. 13, 507 (1945).

[10] F. Franks, Ann. N.Y. Acad. Sci. 125, 277 (1965).

[11] H. Endo, Bull. Chem. Soc. Japan 16, 1586 (1973).

[12] S. Ernst, J. Gliniski, Polish J. Chem. 56, 339 (1982).

[13] S. Ernst, J. Gliński, Mater. Sci. 3, 68 (1977).

[14] M. von Stackelberg, H. Müller, Z. Elektrochem. 58, 25 (1954).

[15] S. Ernst, J. Gliński, B. Jeżowska-Trzebiatowska, Acta Phys. Pol. A 64, 501 (1979).

[16] L. Pauling, in: Hydrogen Bonding, Eds. D. Hadzi, H.W. Thompson, Pergamon, London 1959.

[17] H.S. Frank, A.S. Quist, J. Chem. Phys. 34, 604 (1961).

[18] V.N. Yashkitshev, O.Ya. Samoilov, Zh. Strukt. Khim. 3, 211 (1962).

[19] M.N. Buslayeva, O.Ya. Samoilov, Zh. Strukt. Khim. 4, 502 (1963).

[20] F. Corradini, L. Marcheselli, L. Tassi, G. Tosi, J. Chem. Soc. Faraday Trans. 89, 123 (1993).

[21] E.S. Boek, W.J. Briels, J. Chem. Phys. 98, 1422 (1993).

[22] J.L. Finney, J. Turner, Electrochim. Acta 9, 1183 (1988).

[23] F.S. Frank, F. Franks, J. Chem. Phys. 48, 4746 (1968).

[24] J.A. Schellman, C. R. Trav. Lab. Carlsberg, Ser. Chim. 29, 223 (1955).

[25] G.C. Kreschek, H.A. Scheraga, J. Phys. Chem. 69, 1704 (1965).

[26] R.H. Stokes, Austr. J. Chem. 20, 2087 (1967).

[27] A. Baranowski, K. Jerie, J. Gliński, K. Orzechowski, Acta Phys. Pol. A 88, 59 (1995).

[28] K. Jerie, A. Baranowski, J. Gliński, K. Orzechowski, Acta Phys. Pol. A 85, 563 (1994).

[29] B. Levay, A. Vertes, P. Hautojarvi, J. Phys. Chem. 77, 2229 (1973).

[30] A.P. Buchikhin, V.J. Goldanski, A.O. Tatur, V.P. Shantarovitch, Zh. Eksp. Teor. Fiz. 60, 1136 (1971); S.J. Tao, J. Chem. Phys. 56, 5499 (1972). 